ARTICLE

\title{
UbiD domain dynamics underpins aromatic decarboxylation
}

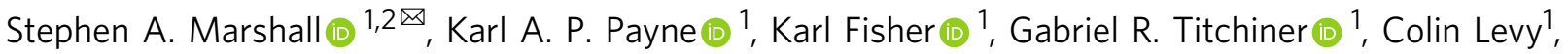 \\ Sam Hay (i) ${ }^{1} \&$ David Leys (iD) ${ }^{1 凶}$
}

The widespread UbiD enzyme family utilises the prFMN cofactor to achieve reversible decarboxylation of acrylic and (hetero)aromatic compounds. The reaction with acrylic compounds based on reversible 1,3-dipolar cycloaddition between substrate and prFMN occurs within the confines of the active site. In contrast, during aromatic acid decarboxylation, substantial rearrangement of the substrate aromatic moiety associated with covalent catalysis presents a molecular dynamic challenge. Here we determine the crystal structures of the multi-subunit vanillic acid decarboxylase $\mathrm{VdcCD}$. We demonstrate that the small $\mathrm{VdcD}$ subunit acts as an allosteric activator of the UbiD-like $\mathrm{VdcC}$. Comparison of distinct $\mathrm{VdcCD}$ structures reveals domain motion of the prFMN-binding domain directly affects active site architecture. Docking of substrate and prFMN-adduct species reveals active site reorganisation coupled to domain motion supports rearrangement of the substrate aromatic moiety. Together with kinetic solvent viscosity effects, this establishes prFMN covalent catalysis of aromatic (de)carboxylation is afforded by UbiD dynamics.

\footnotetext{
${ }^{1}$ Manchester Institute of Biotechnology, University of Manchester, Manchester, UK. ${ }^{2}$ Present address: Chemistry Research Laboratory, University of Oxford, Oxford, UK. ${ }^{凶}$ email: stephen.marshall@chem.ox.ac.uk; david.leys@manchester.ac.uk
} 
$\mathrm{T}$ he processing and utilisation of abundant (hetero)aromatic compounds derived from biomass is a sustainable alternative that reduces reliance on oil derived compounds ${ }^{1-4}$. Recent studies have demonstrated that UbiD enzymes can be used for (hetero)aromatic $\mathrm{C}-\mathrm{H}$ activation at ambient conditions, providing a route to corresponding acids and derivative compounds $^{5-7}$. Studies have also shown the potential of using UbiD enzymes in cascades in the production of cis,cis-muconic acid $^{8,9}$ and in 1,3-butadiene production ${ }^{10}$; both valuable chemicals in the production of synthetic polymers.

The UbiD enzyme family is ubiquitous in microbes and is frequently associated with the flavin prenyltransferase, UbiX. The latter produces the prFMN cofactor required for the UbiDmediated reversible decarboxylation of unsaturated acids (Supplementary Fig. 1) ${ }^{11}$. The UbiD enzyme family can operate on a wide range of unsaturated acrylic, aromatic and heteroaromatic acids, although individual UbiD enzyme substrate specificity has proven to be relatively narrow ${ }^{12}$. Whilst most enzymes function as decarboxylases under physiological conditions, some anaerobic organisms initiate degradation of recalcitrant aromatic compounds such as benzene or naphthalene via UbiD mediated carboxylation ${ }^{13-15}$.

A range of studies with the model system fungal Fdc1 (ferulic acid decarboxylase) have established that a reversible 1,3-dipolar cycloaddition between the acrylic acid dipolarophile and the prFMNiminium azomethine ylide occurs via three distinct intermediates $^{16,17}$ (Fig. 1a). Recent protein engineering studies have established Fdcl can be evolved to accept carboxylated (hetero)aromatic compounds (i.e. where $\mathrm{Ca}$ contributes to the (hetero)aromatic ring), although catalytic rates remain $\operatorname{low}^{5}$. Covalent catalysis with (hetero)aromatic substrates requires transient dearomatisation of the substrate. A range of mechanisms have been proposed for those UbiD enzymes that catalyse decarboxylation of phenolic (Fig. 1b) ${ }^{18}$ and heteroaromatic acids (Fig. 1c) $)^{6,19}$. These all propose formation of a series of covalent adducts between the prFMN C1' and the substrate $\mathrm{Ca}$ (i.e. Int1, 2 and 3). In the case of the Fdc1 mediated reaction with acrylic acids, the structure of various intermediates reveals considerable strain is imposed by the active site, guiding the reaction along ${ }^{17}$. However, in the case of aromatic substrates, it remains unclear how the enzyme active site can accommodate the substantial rearrangement of the aromatic moiety that accompanies the transition between various prFMN-adduct intermediates. Furthermore, the inherent stability of the Int 2 species afforded by rearomatisation of the substrate moiety (as compared to Intl and 3) presents an inherent obstacle to rapid turnover.

It has been suggested that UbiD aromatic acid decarboxylases could accommodate the postulated substrate rearrangement by virtue of domain motion dynamics ${ }^{18,20}$. Indeed, the active site sits at the interface of the prFMN binding and oligomerisation domains, and distinct UbiD enzyme structures fall into either open or closed conformational states (Fig. 1d). However, only the PA0254 (also known as HudA) enzyme has previously been shown to adopt both conformations with the transition between both conformations apparently linked to cofactor binding ${ }^{19}$. Hence, only the dimeric and oxygen-tolerant Fdc1 and PA0254 are representatives of the proposed closed state and are accompanied by substrate or ligand complex structures. In contrast, the hexameric and oxygen-sensitive (hetero)aromatic (de)carboxylases such as $\mathrm{HmfF}$, AroY, and UbiD for which crystal structures are available all adopt open conformations and have yet to yield detailed insights into substrate binding ${ }^{6,18,20}$; the substrates of these enzymes are detailed in Supplementary Table 1.

Furthermore, aside from the obligate requirement for UbiX shared by all UbiDs, a number of the aromatic acid (de)carboxylases are reported to require additional proteins for activity. The vanillic acid decarboxylases for example require a small $(<\sim 10 \mathrm{kDa})$ accessory protein $\mathrm{VdcD}$ for activity, in addition to the UbiD homolog $\mathrm{VdcC}^{21}$. While some UbiD accessory proteins have annotated functions (such as the phosphatase and kinase associated with the phenol phosphate carboxylase enzyme ${ }^{22}$ ), the role of many of the smaller proteins remains unknown. Interestingly, there is no sequence similarity between $\mathrm{VdcD}$, the phenol phosphate carboxylase small subunit (PpcGamma), or LpdD involved in gallic acid decarboxylation in Lactobacillus plantarum ${ }^{23}$.

Here we detail crystal structures of the Sedimentibacter hydroxybenzoicus $\mathrm{VdcCD}$ enzyme and demonstrate that $\mathrm{VdcC}$ forms a tight complex with $\mathrm{VdcD}$, a zinc ribbon protein that allosterically regulates $\mathrm{VdcC}$ activity. A comparison of distinct $\mathrm{VdcCD}$ crystal structures reveal the presence of open and closed states and establishes that $\mathrm{VdcC}$ can undergo a large scale prFMN-binding domain motion, dramatically affecting active site volume. Combined with docking studies and kinetic solvent viscosity effects, our data reveals the structural reorganisation coupled to domain motion supports the rearrangement of the substrate aromatic moiety. This establishes prFMN covalent catalysis of aromatic (de)carboxylation is afforded by UbiD dynamics.

\section{Results}

A VdcCD complex is required for activity. His-tagged versions of the $\mathrm{VdcC}$ proteins from Bacillus subtilis (Bs VdcC) and Sedimentibacter hydroxybenzoicus (ShVdcC; formerly Clostridium hydroxybenzoicum ${ }^{24}$ ) were co-expressed with Pseudomonas aeruginosa UbiX in Escherichia coli (BL21 DE3). However, following purification, the UV-vis spectrum of the isolated preparations did not provide any evidence of cofactor binding. We tested the decarboxylation activity of both $\mathrm{VdcC}$ enzymes reconstituted in vitro with prFMN using vanillic acid and 4-hydroxybenzoic acid (PHB) as substrates. Little to no activity was detected for either VdcC (Fig. 2a), in line with previous reports that $\mathrm{VdcD}$ is essential for activity ${ }^{25,26}$. The corresponding His-tagged $\mathrm{VdcD}$ subunits were therefore expressed in E. coli, and purified VcdD was added to $\mathrm{VdcC}$ preparations prior to in vitro $\mathrm{prFMN}$ reconstitution. When assayed, the $\mathrm{VdcC}+\mathrm{VdcD}$ reconstituted samples demonstrated considerable activity in contrast to isolated $\mathrm{VdcC}$. This demonstrates a strict requirement of both $\mathrm{VdcC}$ and $\mathrm{VdcD}$ for activity. We tested the effect of $\mathrm{VdcC}: \mathrm{VdcD}$ stoichiometry on activity levels of the Sedimentibacter hydroxybenzoicus enzyme. It was found that, while keeping the concentration of $\mathrm{VdcC}$ constant, a sub-stoichiometric $0.2: 1 \mathrm{D}: \mathrm{C}$ ratio led to substantially lower activity when compared with excess D (4:1 D:C ratio) conditions (Fig. 2b). This suggests that $\mathrm{VdcD}$ is likely to form an integral part of the catalytic species, as opposed to a transient role in the VdcC-prFMN complex maturation, for example. To confirm that a VdcCD complex is formed, we respectively co-expressed both $B s \mathrm{VdcC} / \mathrm{D}$ and $\mathrm{ShVdcC} / \mathrm{D}$ enzymes, whereby only one of the subunits was tagged with a hexa-histidine tag. We found that tagging of either $\mathrm{VdcC}$ or D subunit resulted in co-purification of both proteins, yielding the $\mathrm{VdcCD}$ complex in both cases. Unfortunately, despite coexpression of UbiX, this procedure did not yield the holo$\mathrm{VdcCD}$ without in vitro reconstitution.

The VdcCD prFMN cofactor is inactivated by oxygen. To confirm previous reports of $\mathrm{VdcCD}$ oxygen sensitivity, we reconstituted ShVdcCD under anaerobic conditions with prFMNH ${ }_{2}$. The holo-VdcCD was exposed to aerobic conditions and initial decarboxylation activity with PHB was measured at multiple time points over a $90 \mathrm{~min}$ period. Activity decayed with 
a

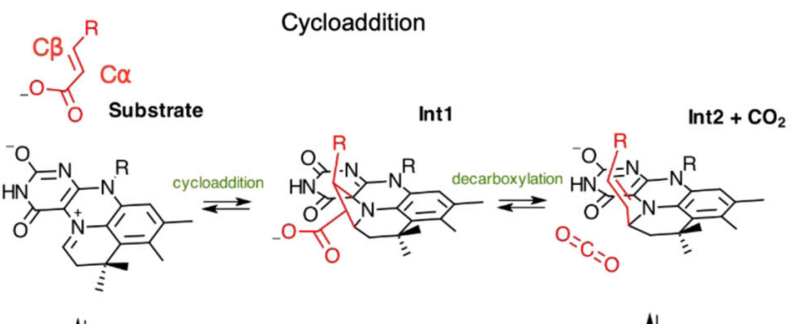

c $\mathrm{C} \beta$

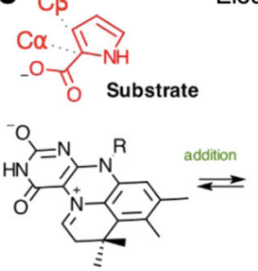

Electrophilic aromatic substitution

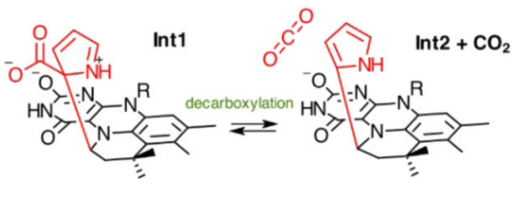

if Glu/ $\mathrm{CO}_{2}$ exchange

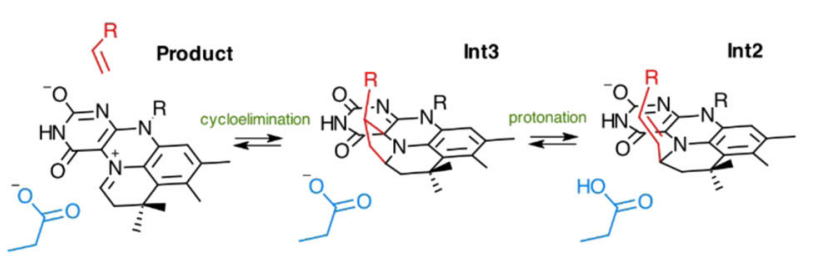

b

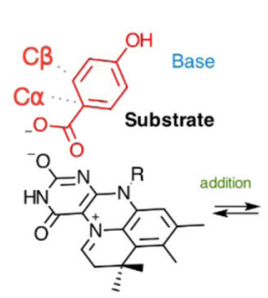

Nucleophilic attack
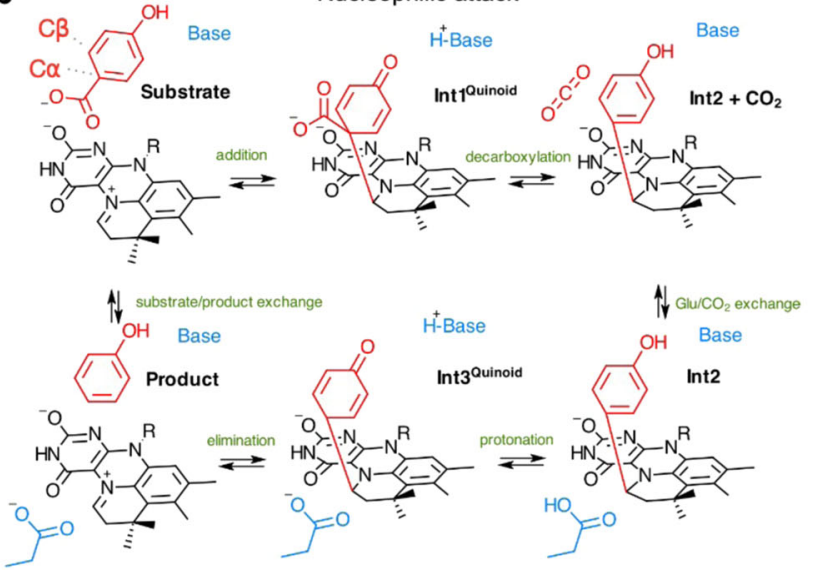

$\uparrow$ substrate/product exchange
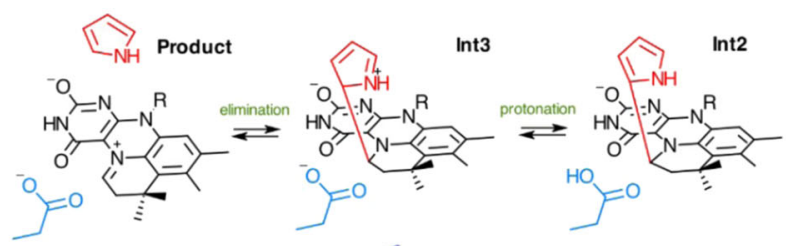

d

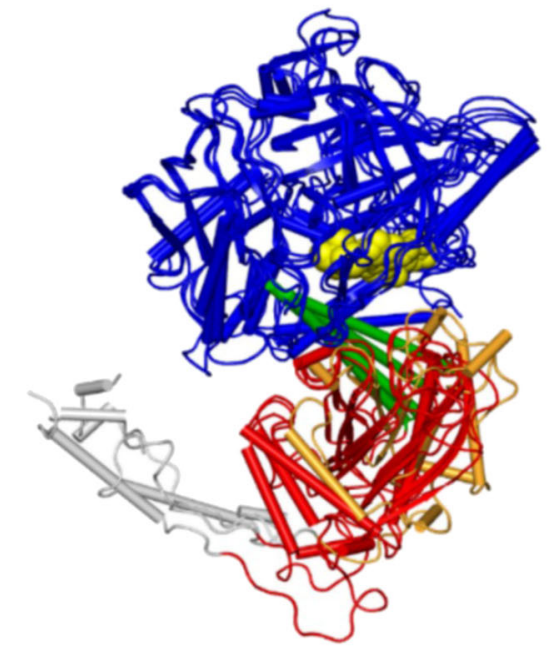

Fig. 1 Mechanism and structures of the UbiD enzyme family. a Proposed 1,3 dipolar cycloaddition Fdc1 mechanism ${ }^{16,17}$. b A substrate nucleophilic attack mechanism proposed for phenolic substrates ${ }^{18}$. c Electrophilic aromatic substitution mechanism postulated for PA0254-mediated decarboxylation of pyrrole-2-carboxylate ${ }^{19}$. All mechanisms share a common Int2 intermediate with an sp ${ }^{2}$ hybridized substrate $\mathrm{C} \alpha$. Transient dearomatisation occurring for Int1 and Int3 species presents a significant energy barrier in each case. prFMN-R = phosphoribityl moiety (omitted for clarity) d Overlay of UbiD monomers anchored on the prFMN binding domain (in blue). The oligomerisation domains of Fdc and PA0254 are shown in orange (representing a closed conformation), while UbiD, HmfF and AroY are shown in open states (in red). The C-terminal helix is shown in grey. The hinge region is located within a helix (in green) connecting the oligomerisation and prFMN binding domains, close to bound cofactor (in yellow spheres).

an approximate half-life of 40 minutes (Fig. 2c). Following $90 \mathrm{~min}$ oxygen exposure, addition of 2-fold excess of prFMNH 2 led to significant recovery of activity and demonstrates that the oxygen inactivation of $\mathrm{VdcCD}$ occurs at the level of the cofactor. In the case of phthaloyl co-A decarboxylase, it is postulated oxidation of the prFMN associated metal ion (Fe(II) in this case) leads to a simple loss of the cofactor ${ }^{27}$. However, it is unlikely oxidation of $\mathrm{Mn}^{2+}$ underpins oxygen inactivation of $\mathrm{VdcCD}$ as the addition of cofactor alone (without additional $\mathrm{Mn}^{2+}$ ) is sufficient in restoring activity.

VdcCD crystal structure reveals a tight association between both subunits. The apo-ShVdcCD complex was crystallised under a number of conditions, with different crystal forms yielding diffraction data at a resolution ranging from $2.2 \AA$ to 3.4 A. Despite multiple attempts, no crystals of individual subunits or the holo-ShVdcCD complex could be obtained. The ShVdcCD structures were solved using molecular replacement with individual domains of the E. coli (K12) UbiD as the search models (PDB code 5M1C). The ShVdcCD crystal structures contain a ShVdcC hexamer decorated with 6 small ShVdcD subunits (Fig. 3a). The $S h \mathrm{VdcD}$ was found to bind a zinc ion in a tetrahedral coordination with four cysteine residues ( $\mathrm{ShVdcD}$ numbering C3, C6, C28 and C31) (Fig. 3b). The 4 Cys residues are arranged in two CXXC knuckle motifs, an arrangement common for zinc ribbon proteins ${ }^{28}$. Indeed, $S h \mathrm{VdcD}$ forms a three stranded, antiparallel $\beta$-sheet, and shares structural similarities with the zinc ribbon protein TF-IIS as identified by PDBeFold ${ }^{29}$ (RMSD - $1.77 \AA$ over 37 residues - PDB accession$1 \mathrm{TFI}^{30}$ ). Both the $\beta$-sheet of ShVdcD as well as the proline rich $\mathrm{C}$-terminal tail region interact with the surface of $S h \mathrm{VdcC}$. The ShVdcC:D interface was analysed by PISA $^{31}$ and was shown to have an average area of $1661 \AA^{2}$, involving 40 residues of $S h \mathrm{VdcD}$ (59\% of total residues), and 50 residues of $\operatorname{ShVdcC}(10.5 \%$ of total residues). In addition to a number of hydrogen bonds and van der Waals interactions, intersubunit salt bridges were identified with ShVdcD R12, R36 and E39 forming interactions with ShVdcC D173, E31 and R133. Sequence analysis of multiple VdcD show that the two CXXC motifs are conserved, therefore $\mathrm{Zn}^{2+}$ binding is likely an essential structural motif to maintain the sheet formation and interaction with $S h \mathrm{VdcC}$. The location of the ShVdcC: ShVdcD interface suggests that the allosteric effect is based on maintaining the structure of the key catalytic ERE 


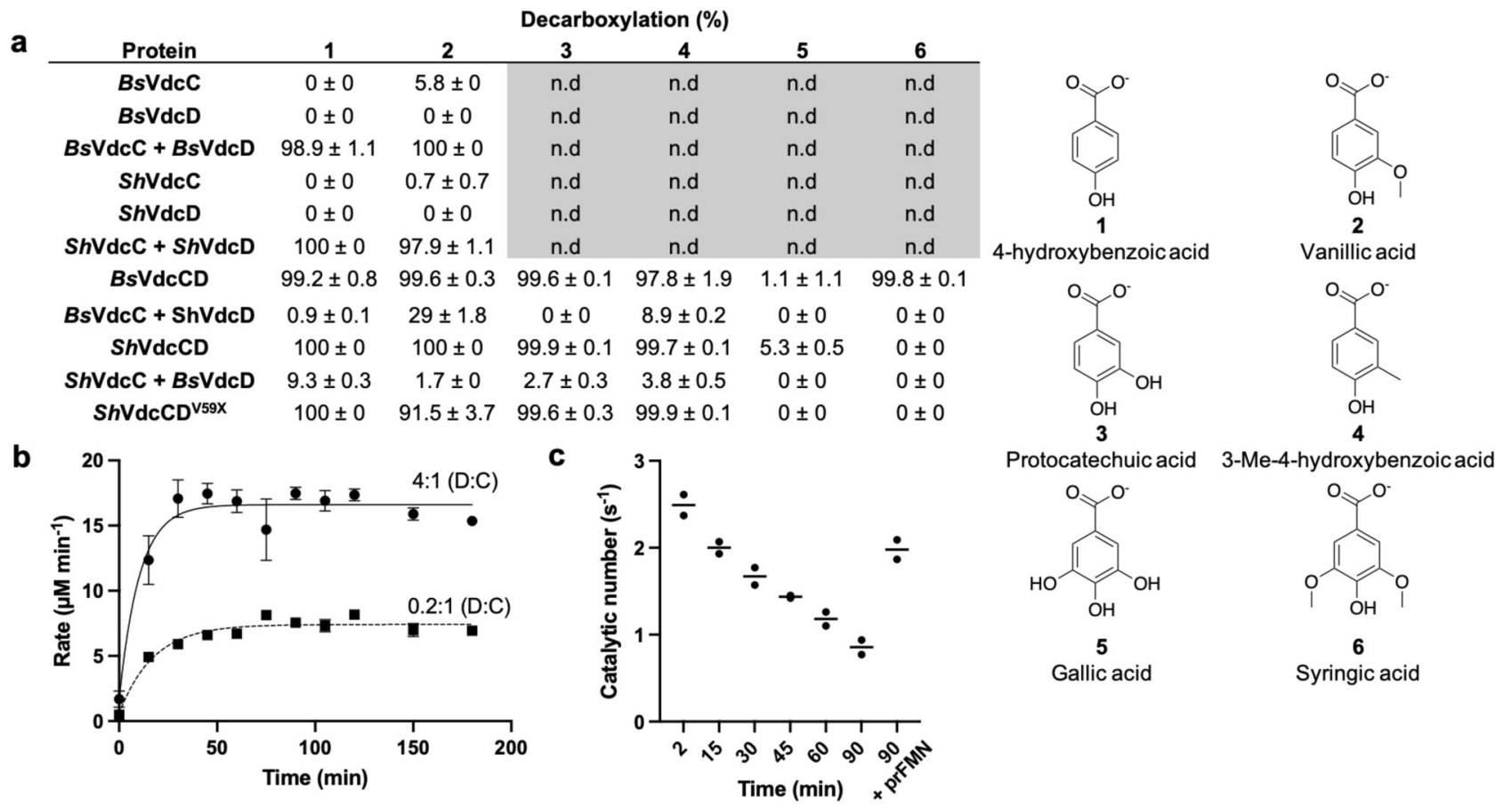

Fig. 2 Activity of Vdc proteins. a Activity of reconstituted Vdc proteins. Significant activity is only seen when both $\mathrm{VdcC}$ and $\mathrm{VdcD}$ are present. Protein obtained through co-purification is denoted as $\mathrm{VdcCD}$. Where proteins were purified separately and combined in vitro, the label $\mathrm{VdcC}+\mathrm{VdcD}$ is used. Substrate specificity of $\mathrm{VdcCD}$ enzymes from Bacillus subtilis and Sedimentibacter hydroxybenziocus show similar substrate ranges, with the exception of syringic acid. Non-cognate $\mathrm{VdcD}$ can be used for the limited activation of $\mathrm{VdcC}$. Deletion of the proline rich tail of $\mathrm{VdcD}$ has little effect on the activity of $\mathrm{VdcCD}$. Data shown as mean $\pm \mathrm{SEM} n=2$ independent replicates. $\mathbf{b}$ Effect of $\mathrm{VdcC}: \mathrm{VdcD}$ ratio on decarboxylation rates of $100 \mu \mathrm{M}$ PHB. A sub-equimolar ratio results in slower decarboxylation of PHB, suggesting a non-transient interaction between $\mathrm{VdcC}$ and $\mathrm{VdcD}$. Data are represented by mean $\pm \mathrm{SEM}, n=3$ technical replicates. Data trends seen in three independent replicates. c Decrease in activity of ShVdcCD with $100 \mu M$ PHB in aerobic conditions. Addition of prFMNH 2 partially restores activity, all data points shown, bar represents mean. Data from two independent replicates.
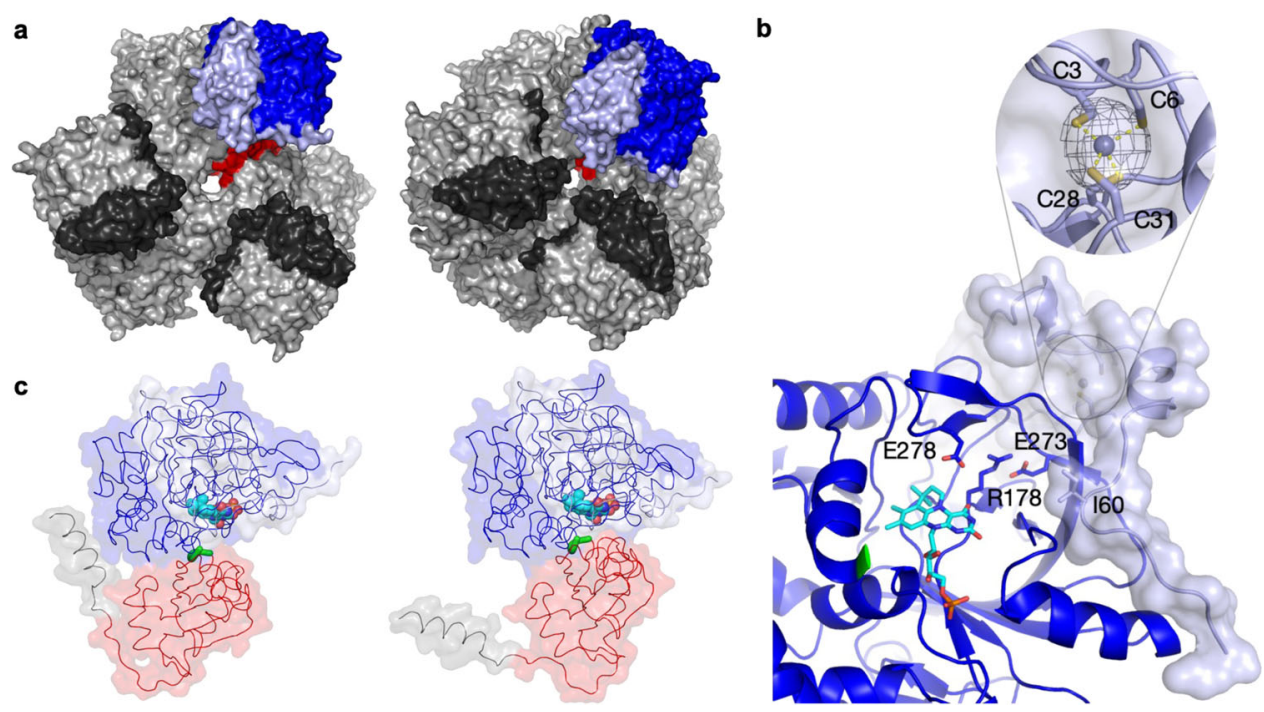

Fig. 3 Structures of VdcCD complexes in open and closed states. a Left - Open hexamer of ShVdcCD, Right - closed hexamer of ShVdcCD. A single monomer of ShVdcC is coloured by domain (red - oligomerisation domain, blue - prFMN binding domain) with ShVdcD in light blue. All other ShVdcC in the complex are shown in light grey, with $S h V d c D$ shown in dark grey. $\mathbf{b}$ Interaction of $S h V d c C$ and $S h V d c D$ showing the proximity of $V d c D$ binding to the $\mathrm{VdcC}$ active site. $\mathrm{VdcD}$ interacts with loops containing the key ERE residues which are a key triad for catalysis, VdcD likely serves to maintain the positioning of these residues. prFMN (in cyan) is modelled in the active site based on a structural alignment with holo-Fdc1 (4ZA4). Inset shows the $\mathrm{VdcD}$ tetrahedral binding of $\mathrm{Zn}^{2+}$ by four conserved cysteine residues. Polder map corresponding to $\mathrm{Zn}^{2+}$ contoured at 5 sigma. $\mathbf{c}$ Dyndom analysis reveals a $32^{\circ}$ rotation at the T325 and S326 hinge region (in green) connects the open (left) and closed states (right). Other domains coloured as in $\mathbf{a}$, with the C-terminal helix coloured in grey. 
motif $^{32}$ (Fig. 3b). Intriguingly, despite the relatively low sequence identity of $39 \%$ ( $48 \%$ similarity) between the $\mathrm{VdcD}$ subunits, limited activation of the $C$ subunit could be achieved with the addition of a non-cognate $\mathrm{D}$ subunit (Fig. 2a).

Distinct VdcCD crystal structures reveal prFMN domain motion. A comparison of the $S h \mathrm{VdcC}$ monomers across the ShVdcCD crystal structures obtained reveals that the prFMN binding domain adopts different conformations with respect to the oligomerisation domain, confirming the existence of distinct open and closed states (Supplementary movie 1 illustrates the motion between the open and closed hexamers). The distinct states occur independent of both prFMN cofactor and $\mathrm{VdcD}$ binding (both states occur for the apo-VdcCD complex), in contrast to the apo-open and holo-closed PA0254 structures where domain motion appears linked to cofactor binding ${ }^{19}$. $S h \mathrm{VdcCD}$ structures thus allow for detailed inspection of the transition between the two states. An analysis of the domain motion using DynDom ${ }^{33}$ identifies this as a hinge motion, with the point of rotation located at T325 and S326, located in an ahelix connecting the oligomerisation and prFMN binding domains (Fig. 3c). This helix has been previously postulated as the point of flexibility in E. coli $\mathrm{UbiD}^{20}$ and $\mathrm{AroY}^{18}$ on the basis of the small hinge motions observed by comparing various open state monomers. In the case of PA0254, overlays of the respective apo- and holo-structures also requires a similar hinge motion ${ }^{19}$. In $\mathrm{VdcCD}$, the hinge rotation angle is approximately $32^{\circ}$, with only a negligible translation of the domains $(<1 \AA)$. The number of interdomain contacts of the closed state outnumber those of the open state, with 11 more paired residue contacts, the majority occurring close to the active site (Supplementary Fig. 2).

The distance between the centre-of-mass (calculated by the CALCOM server ${ }^{34}$ ) of residues R168/173 (ShVdcC/Fdc numbering) on the prFMN binding domain and L425/439 on the oligomerisation domain can be used as a simple indicator of open or closed conformation (Supplementary Fig. 3a). These residues are highly conserved in the UbiD family and have been demonstrated to be essential in catalysis by $\mathrm{Fdcl}^{17,32}$. In the open ShVdcCD the mean distances $( \pm \mathrm{SD})$ are $14.5 \pm 0.1 \AA$ (P1 crystal form) and $14.1 \pm 1.4 \AA$ ( $22_{1} 2_{1} 2_{1}$ crystal form), compared to $9.3 \pm 0.1 \AA$ for the closed (F222) form. When determining the distance between the corresponding residues in other UbiD structures, only Fdc1 and holo-PA0254 crystal structures clearly correlate to a closed state ( $9.8 \AA$ and $10.1 \pm 0.1 \AA$ respectively), while distances for AroY, UbiD, HmfF and TtnD are distributed over a wide range corresponding to the open conformation (Supplementary Fig. 3b).

Modelling of holo-VdcCD complexes. An overlay of the structure of a closed ShVdcCD structure with the model UbiD enzyme Aspergillus niger Fdc1 (PDB 4ZA4), allows for the ShVdcCD prFMN binding site to be easily located and active site residues identified. Docking prFMN to the closed ShVdcCD structure using Molsoft ICM Pro (version 3.9-2b) and Rosetta Ligand Docking ${ }^{35,36}$ resulted in a similar binding conformation (Fig. 4a). All residues implicated in catalysis, including the UbiD-conserved E(D)RE motif integral for activity, are present in ShVdcCD.

We were able to assess binding of vanillic acid in ShVdcCD by superimposition with the naphthoic acid (6TIB) AnFdc complex ${ }^{5}$ (Supplementary Fig. 4). The proposed substrate binding mode suggests $\mathrm{H} 349$ and Y381 position the substrate of the $\mathrm{C} \alpha-\mathrm{C} \beta$ bond in ideal geometry in relation to $\mathrm{C1}^{\prime}$ and $\mathrm{C} 4 \mathrm{a}$ and could support substrate phenolate formation during catalysis. To assess the impact of domain motion on catalysis, we assessed the binding of substrate and prFMN adducts to intermediate structures along the trajectory of closure (generated using Chimera Morph between the open and closed states ${ }^{37}$ ) by docking using AutoDock Vina ${ }^{38}$. The morph model used for docking was validated by assessing the domain motion using low-frequency normal mode analysis performed with elNémo ${ }^{39}$ (Supplementary movie 2), which shows similar domain motions. The catalytic glutamate residue (E278) adopts a range of conformations in other UbiD enzymes, adapting to the presence and nature of ligands bound in the active site. For this reason, the E278 rotamer was altered to avoid clashes when docking carboxylated compounds. Docking $\mathrm{PHB}$ to the holo-VdcCD reveals intermediate states (i.e. R-L distance $=11.6 \AA$ ) result in lowest energy, whereas docking vanillic acid reveal that R-L distances of $13.4 \AA$ show the lowest energy (Fig. 4b). However, the relative position of the substrate with respect to prFMN is subtly altered by the domain closure, and resembles previously determined Fdc1 ligand complexes most closely at R-L distances of $10.4 \AA$ and $10.0 \AA$ for PHB and vanillic acid respectively (Fig. 4c). Hence, domain closure likely minimizes the substrate $\mathrm{Ca} / \mathrm{prFMN} \mathrm{C1}$ ' distance prior to Int1 formation.

DFT generated models of the PHB adducts Int 2 and Int 3 Quinoid (Int1Quinoid spontaneously decarboxylates) were docked to apo$\mathrm{VdcCD}$ structures along the domain closure trajectory. The Int2 complex has the lowest energy for the longer R-L distances, while Int3 Quinoid complex displays an energy minimum at R-L distance $\sim 10 \AA$ (Figs. $4 \mathrm{~b}$ and 5). Hence, docking analysis suggests domain motion accompanies the Int $\mathbf{1 / 3}$ Quinoid transition to Int2. Furthermore, it is possible domain closure leads to a strained conformation of Int2, thus assisting conversion to Int1/3 Quinoid and ultimately product formation.

Kinetic solvent viscosity effects support a VdcCD dynamic model. To determine whether catalysis is coupled to proposed domain motions, we determined the kinetic parameters of $\mathrm{PHB}$ decarboxylation by $\mathrm{ShVdcCD}$ in the presence of viscosogens. It was found that $\mathrm{k}_{\mathrm{cat}}$ app was drastically reduced in $45 \%(\mathrm{v} / \mathrm{v})$ glycerol or $40 \%(\mathrm{w} / \mathrm{v})$ sucrose conditions (Fig. $4 \mathrm{~d})$. In contrast, the $\mathrm{K}_{\mathrm{m}}$ app for PHB is not significantly impacted within the error of the data, suggesting neither microviscosogen acts as competitive inhibitor. The decrease of $k_{c a t}$ app with relative viscosity ( $\eta$ ) supports the proposed $\mathrm{VdcCD}$ dynamic model for catalysis with increased viscosity affecting domain motion ${ }^{40}$.

The VdcD unit does not directly contribute to catalysis. Only a single $S h \mathrm{VdcD}$ residue (I60) can be considered to contribute to the active site architecture (Figs. $3 \mathrm{~b}$ and $4 \mathrm{a}$ ). This residue is located on a conserved proline rich tail located at the $S h \mathrm{VdcCD}$ interface. We sought to investigate the role of this C-terminal tail by deleting ShVdcD residues 59-68 (VIPPIPPLKK). The resulting ShVdcCD V59X complex was determined to be active, with decarboxylation yields only slightly diminished (i.e. $<10 \%$ reduction in yield) compared to those of the wild type (Fig. 2a). ShVdcCD ${ }^{\mathrm{V} 59 \mathrm{X}}$ crystallised in a different space group $\left(\mathrm{P} 22_{1} 2_{1}\right)$ from the WT open (P1) and closed (F222) forms. The ShVdcCDV59X P2 ${ }_{1} 22_{1}$ structure contains a single heterododecamer in the asymmetric unit, with the majority of individual ShVdcC monomers adopting an open form. A comparison to the wild type structure shows little difference in the relative positions of the active site residues, with the exception of a minor shift in residues directly adjacent to the deleted tail region. The observation that $S h \mathrm{VdcD}$ does not appear to directly contribute to the active site architecture supports the notion that its role is mainly in the stabilisation of the catalytic ERE containing loop regions at the $S h \mathrm{VdcCD}$ interface. 
a

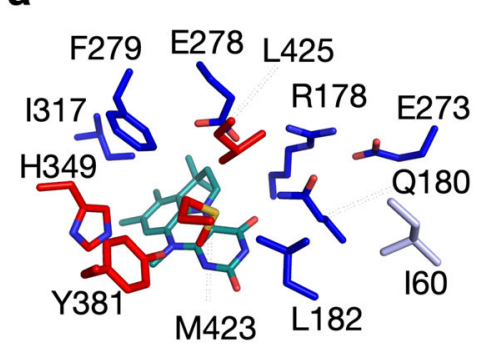

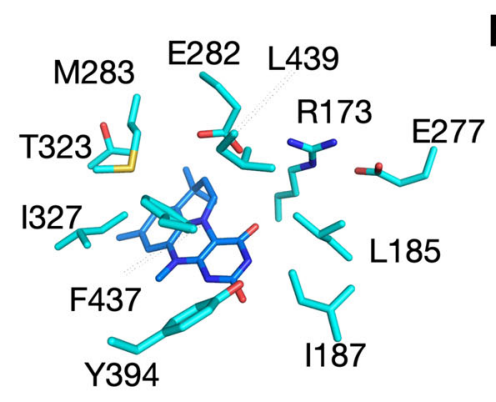

C
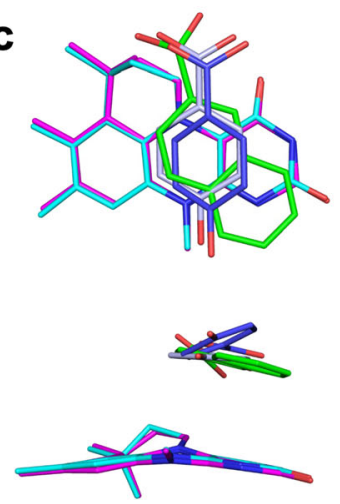
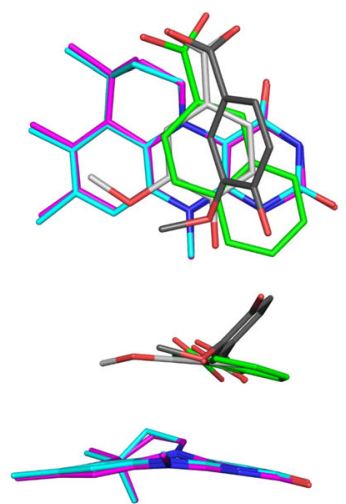

d
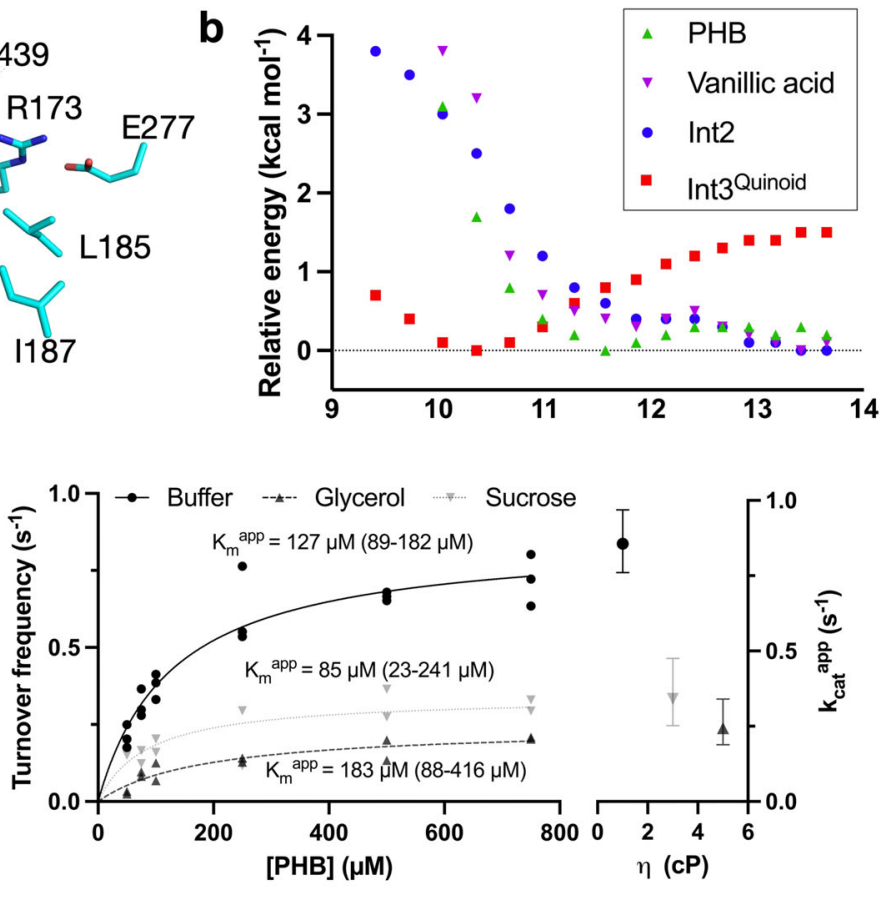

Fig. 4 Active site architecture and the effect of domain motion. a Left: Active site composition of closed ShVdcCD shows limited direct contribution of $\mathrm{VdcD}(160)$ to the active site architecture. Residues coloured by domain as in Fig. 3. prFMN modelled in dark green from Rosetta Ligand Docking. Right: Juxtaposition of Fdc1 active site shows similar overall architecture to the active site, with residues key for Fdc1 catalysis (E282, R173, E277, L439) conserved between the enzymes. $\mathbf{b}$ Energy profile of docking models along the trajectory of domain closure. Energy minima of PHB and vanillic acid binding to $\mathrm{VdcCD}$ with previously docked prFMN are found when R-L distances are 11.6 and $13.4 \AA$ respectively. Int2 shows lowest binding energies to most open models, whereas Int3 $\mathbf{Q}^{\text {Quinoid }}$ shows lowest binding energies at an R-L distance of $10 \AA$. c Modelled substrate binding modes identified by docking with AutoDock Vina. Bottom images $90^{\circ}$ rotation about $X$ axis. Cyan prFMN - Fdc1 (6TIB), magenta prFMN - ShVdcCD Rosetta ligand docking to closed structure. Left: PHB lowest energy binding (dark blue) found when R-L distance is $11.6 \AA$, and binding mode (light blue) most similar to naphthoic acid (green) in Fdc1 found when R-L distance is $10.4 \AA$. Right: Vanillic acid lowest energy binding (dark grey) found when R-L distance is $13.4 \AA$, and binding mode (light grey) most similar to naphthoic acid (green) in Fdc1 found when R-L distance is $10 \AA$. $\mathbf{d}$ Kinetic solvent viscosity effects observed for PHB decarboxylation performed in $45 \%$ glycerol or $40 \%$ sucrose compared to buffer without viscosogen. Non-linear regression fit to Michaelis-Menten kinetics using Prism 9. $\mathrm{K}_{\mathrm{m}}$ app does not vary significantly between samples (within $95 \%$ confidence limits). $n=3$ (buffer) $n=2$ (glycerol and sucrose) technical replicates, all replicates shown. Similar trends were seen in two independent replicates. The inset shows calculated $\mathrm{k}_{\text {cat }}{ }^{\text {app }}$ plotted against relative viscosity $(\eta)$ revealing a significant decrease in catalytic rate constant with increasing viscosity. Values of $\eta$ estimated from literature ${ }^{51}$. Data shown as mean, with error bars showing $95 \%$ confidence limits.

VdcCD substrate specificity. The substrate scope of both $B s \mathrm{VdcCD}$ and ShVdcCD were determined by endpoint assays, incubating the enzymes overnight with $5 \mathrm{mM}$ substrate prior to quenching the reaction. The substrate scope of these enzymes is shown to be similar, as expected given the $58 \%$ identity between $S h \mathrm{VdcCD}$ and BsVdcCD (Fig. 2a). Notable exceptions are syringic acid, for which product formation could only be detected in the presence of $B s \mathrm{VdcCD}$, and gallic acid which only supported minor activity with ShVdcCD. This can be explained by consideration of the respective active site structures. The BsVdcCD active site volume is larger due to the substitution of Met 423 for Leu419 and Leu182 for Val179 on the C subunit, as well as the D subunit Ile60 replaced by Val67 (Supplementary Fig. 5). This presumably allows for correct positioning of the second methoxy group of syringic acid. An L182V mutant of ShVdcCD was found to be capable of syringic acid decarboxylation with $6.5 \pm 0.6 \%$ turnover compared to no turnover seen in the wild type protein, thus further validating the proposed substrate binding model.

\section{Discussion}

The covalent catalysis used by the prFMN-dependent enzymes presents a range of challenges to ensure rapid turnover. In the case of the fungal Fdc1 enzyme, considerable strain imposed by the rigid active site for various intermediates along the pathway has been observed ${ }^{17}$. This is proposed to support the reversible nature of the 1,3-dipolar cycloaddition process and avoid the formation of dead-end adduct species. While these are avoided for the relatively large substrates, dead-end adducts are formed with the considerably smaller crotonic acid, presumably because the enzyme is unable to exert strain in this case. The aromatic acid UbiD decarboxylases are faced with a range of challenges. These including the need to surmount the inherent aromatic stability of the substrate, the requirement to accommodate the relatively large motion of the substrate aromatic moiety upon progression through the various prFMN-covalent adducts, as well as the ability to manipulate the internal thermodynamics of the reaction to avoid highly stable aromatic Int 2 species following decarboxylation/deprotonation.

We demonstrate that UbiD-enzymes have the scope to adapt the shape of the active site via domain motion by determining structures of open and closed states for the vanillic acid decarboxylase VdcCD (Fig. 5). While the VdcCD closed state resembles the conformation observed for fungal $\mathrm{Fdc1}$, the open state resembles the majority of UbiD structures hitherto determined. Modelling of the VdcCD domain closure trajectory and assessing the binding of substrate and intermediates reveals an intermediate conformation binds substrate most favourably. 


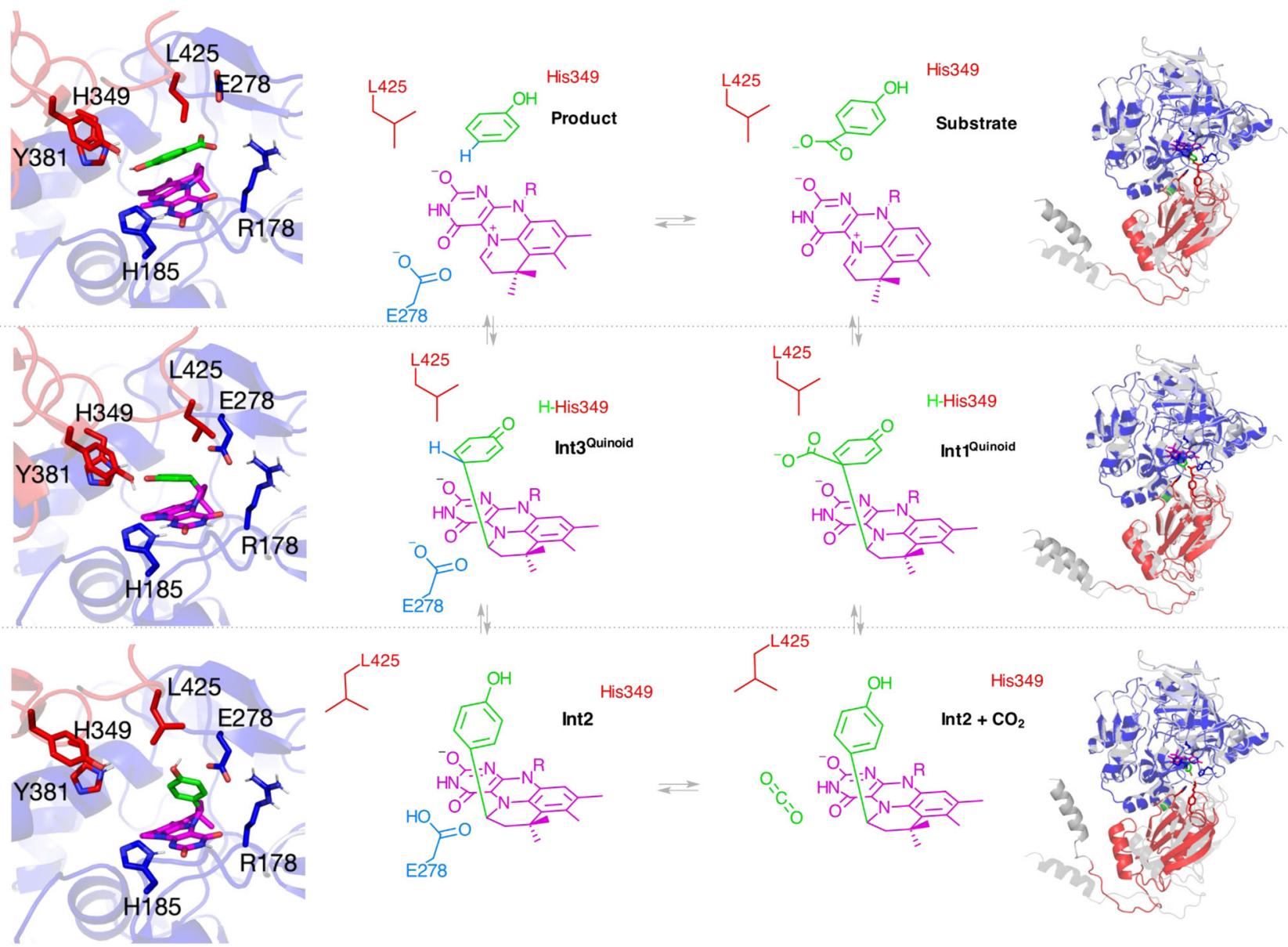

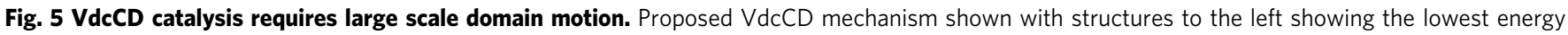
configuration of substrate and intermediates bound in ShVdcCD active site (prFMN depicted in magenta, and substrate/adducts depicted in green). The structures to the right show the corresponding monomer conformation, overlayed on the closed structure (in translucent grey). Supplementary movie 3 illustrates the domain motions seen between intermediates.

Further domain closure effectively brings prFMN and substrate together and favours binding of the Int1/Int3 Quinoid states. The $s p^{3}$ to $s p^{2}$ transition of the substrate $\mathrm{Ca}$ concomitant with decarboxylation can be accommodated by opening of the active site. Re-aromatisation thus drives formation of the highly stable Int2 species and domain opening. If the energetics of domain closure are such that the open-closed equilibrium is poised towards the closed state, this would provide a means to exert strain on Int 2 by domain closure and avoid formation of dead-end intermediates. The kinetic solvent viscosity effects observed for PHB decarboxylation align with the proposed dynamic model for $\mathrm{VdcCD}$ catalysis. It is likely that (de)carboxylation of other aromatic acids, although not necessarily progressing through a quinoid intermediate, requires similar active site mobility to accompany formation of and interconversion between covalent prFMN adducts.

UbiD enzyme activity is often found to be oxygen sensitive, with the exception of family members acting upon acrylic (Fdc1) or heteroaromatic substrates (PA0254). Both Fdc1 and PA0254 form dimers ${ }^{16,41}$, in contrast to the more frequently observed hexameric nature of the aromatic acid decarboxylases ${ }^{18,20}$. Furthermore, there is little evidence that large scale domain motion occurs for the dimeric holo-enzymes or is involved in catalysis. In contrast, based on our work with $\mathrm{VdcCD}$, it appears domain motion is integral to enzyme turnover for aromatic acid (de) carboxylation. It is possible the open state preferentially populated by the hexameric holo-enzymes is markedly different in oxygen sensitivity compared to the closed state readily observed for the dimeric UbiD enzymes. Furthermore, the prFMN reactivity is likely to be modulated by the protein environment, in particular residues that interact with the $\mathrm{O} 1$ position. In the case of the oxygen sensitive UbiD enzymes, these include positively charged residues (i.e. Arg/Lys/His), while for those apparently unaffected by oxygen, hydrogen bonding partners are Asn or Gln residues. This difference in active site composition may be responsible for the ability to decarboxylate acids fused directly to the aromatic ring, at the expense of stability in aerobic conditions. Indeed, evolved variants of the oxygen tolerant Fdc1 have been reported to undergo oxygen mediated inactivation ${ }^{10}$, suggesting active site properties influence oxygen sensitivity.

The rational engineering of $S h \mathrm{VdcCD}$ to accept syringic acid using $\mathrm{L} 182 \mathrm{~V}$ provides further evidence that UbiD enzymes can be evolved, but also suggests further studies will need to take into account the effect mutations might have on the protein dynamical behaviour. In conclusion, domain dynamics coupled to catalysis provides a solution to some of the challenges faced by aromatic acid UbiD decarboxylases. Appropriate choreography between domain dynamics and catalysis is likely essential to achieve rapid turnover, by accommodating large scale motion of the substrate aromatic moiety and manipulating the internal thermodynamics to avoid the pitfall of an excessively stable Int 2 species. This certainly would be the case of very challenging substrates such as benzene or naphthalene $e^{14,15}$. Indeed, while rational engineering of the dimeric Fdcl has led to some success in expanding the substrate scope, Fdcl mediated naphthoic acid decarboxylation 
remains very slow ${ }^{5}$. It seems likely the lack of significant domain motion in Fdcl will need to be addressed in order to achieve improved catalytic rates.

\section{Methods}

Cloning, mutagenesis, and cell growth. Sedimentibacter hydroxybenzoicus $v d c C$ and $v d c D$ genes were codon optimized for E. coli expression and synthesised by GeneArt (ThermoFisher), Bacillus subtilis $v d c C$ and $v d c D$ were codon optimized for E. coli expression and synthesised by GenScript. C-terminal His tag constructs of the $\mathrm{C}$ subunits were made in pET30a between $\mathrm{NdeI}$ and XhoI restriction sites. $\mathrm{N}$-terminal His tag constructs of D subunits were made in pET28a between NdeI and XhoI restriction sites. Untagged constructs of both C and D subunits were made in pET21a between NdeI and XhoI. A construct of untagged PaUbiX in pCDF-Duet was used ${ }^{6}$. These constructs were made to allow different combinations of vectors to be used with different antibiotic resistances to maintain selection pressures.

The genes were amplified using the PCR primers detailed in Supplementary Table 2.

The empty vectors were cut using restriction enzymes purchased from NEB. InFusion (Takara) ligation independent cloning was used to insert the fragments into cut vectors. Stellar cells (Takara) were transformed with the product, and insertion was confirmed by sequencing (Eurofins) following MiniPrep (Qiagen) of overnight cultures of single colonies.

Mutants were produced by using Q5 mutagenesis (NEB) as per the manufacturers protocols, using the primers in Supplementary Table 2. Mutation was confirmed by sequencing.

BL21(DE3) cells (NEB) were transformed with plasmid in the combinations denoted in Supplementary Table 3.

BL21(DE3) strains were grown at $37^{\circ} \mathrm{C}, 180 \mathrm{rpm}$ shaking in $1 \mathrm{~L}$ conical flasks of Terrific Broth (Formedium) with $0.4 \%$ (v/v) glycerol added, along with $50 \mathrm{mg} \mathrm{L}^{-1}$ of the appropriate antibiotic(s). Cells were induced with $0.25 \mathrm{mM}$ IPTG when $\mathrm{OD}_{600 n m}$ reached 0.8 , at which point the temperature was reduced to $18^{\circ} \mathrm{C}$ overnight. Cells were harvested after typically $18 \mathrm{~h}$ of induction by centrifugation at $7000 \times g$ for $10 \mathrm{~min}$ in a Beckman JLA8.1000 rotor.

Purification. Cells were resuspended in buffer A (50 mM Hepes, $300 \mathrm{mM} \mathrm{NaCl}$, $\mathrm{pH}$ 7.5) with SigmaFAST EDTA free protease inhibitor cocktail tablet added, $0.1 \mathrm{mg} \mathrm{mL}^{-1}$ DNase and RNase were both added to the cell suspension (all Sigma). Cells were lysed using a Constant Systems cell disruptor at $20 \mathrm{kPsi}$. Lysate was clarified by centrifugation at an average $184,000 \times g$ for $1 \mathrm{~h}$ in a Beckman Type 50.2Ti rotor. Using an AKTA Pure (GE Healthcare), the lysate was applied to a pre-equilibrated $5 \mathrm{~mL}$ HisTrap-HP (GE Healthcare) column and was washed with $5 \mathrm{CV}$ buffer A. A gradient of buffer A to buffer A containing $250 \mathrm{mM}$ imidazole was run over $30 \mathrm{CV}$ and collected in $1 \mathrm{~mL}$ fractions. Proteins eluted in a single peak and were run on an SDS PAGE gel for confirmation of purity. Fractions containing the desired protein were concentrated using Sartorius Vivaspin concentrators, and subjected to gel filtration using a Superdex 200 10/300 GL column (GE Healthcare), in $20 \mathrm{mM}$ Hepes, $150 \mathrm{mM} \mathrm{NaCl}, \mathrm{pH} 7.5$ to remove any excess $\mathrm{VdcD}$ and to ensure 1:1 stoichiometry.

For protein prepared for crystallisation, the $S h \mathrm{VdcCD}^{\mathrm{N}} / S h \mathrm{VdcCD} V 59 \mathrm{X}$ complexes were further purified after IMAC. The fractions from Ni-IMAC were pooled and concentrated, prior to a 6 -fold dilution using $50 \mathrm{mM}$ Hepes, $\mathrm{pH} 7.5$ to dilute $\mathrm{NaCl}$ to $50 \mathrm{mM}$. Using an AKTA Pure (GE Healthcare), the complex was applied to a $5 \mathrm{~mL}$ HiTrapQ-HP (GE Healthcare) column and was washed with 5 CV $50 \mathrm{mM}$ Hepes, $50 \mathrm{mM} \mathrm{NaCl}$, pH 7.5. A gradient of $50 \mathrm{mM}-1 \mathrm{M} \mathrm{NaCl}$ was run over $30 \mathrm{CV}$, in a constant buffer of $50 \mathrm{mM}$ Hepes $\mathrm{pH}$ 7.5. The peak containing the complex was pooled, concentrated, and subjected to gel filtration using a Superdex 200 10/300 GL column (GE Healthcare), in $20 \mathrm{mM}$ Hepes, $150 \mathrm{mM}$ $\mathrm{NaCl}, \mathrm{pH}$ 7.5. The protein was concentrated in Vivaspin concentrators (Sartorius) and crystal trials were immediately performed.

Protein concentrations were estimated using $\mathrm{A}_{280 \mathrm{~nm}}$ extinction coefficients calculated by the ExPASy ProtParam tool: $\operatorname{ShVdcC} \varepsilon=57300 \mathrm{M}^{-1} \mathrm{~cm}^{-1}, B s \mathrm{VdcC}$ $\varepsilon=58790 \mathrm{M}^{-1} \mathrm{~cm}^{-1}$, ShVdcD, ShVdcDV59X, and $B s \mathrm{VdcD} \varepsilon=13980 \mathrm{M}^{-1} \mathrm{~cm}^{-1}$, $\operatorname{ShVdcCD} \varepsilon=71280 \mathrm{M}^{-1} \mathrm{~cm}^{-1}, B s \mathrm{VdcCD} \varepsilon=72770 \mathrm{M}^{-1} \mathrm{~cm}^{-1}$.

HPLC activity assays. All HPLC assays were prepared in an anoxic chamber (Belle Technologies) in a nitrogen atmosphere. prFMN was made as per previous protocols $^{42} ; 50 \mu \mathrm{M}$ UbiX $^{11}, 5 \mu \mathrm{M}$ Fre reductase ${ }^{43}, 1 \mathrm{mM}$ FMN, $5 \mathrm{mM}$ DMAP, and $5 \mathrm{mM}$ NADH were combined in an anaerobic chamber and left for $2 \mathrm{~h}$ to allow prFMN production before filtration through a $10 \mathrm{kDa}$ MWCO centrifugal concentrator (Sartorius) to remove UbiX and Fre proteins prior to addition to Vdc assays.

Initial assays were performed with substrates (4-hydroxybenzoic acid (PHB) and vanillic acid) dissolved to $10 \mathrm{mM}$ in $100 \mathrm{mM} \mathrm{KP} i$, pH 6; substrates were not purged of oxygen before introduction to the anaerobic chamber. $\mathrm{VdcC}, \mathrm{VdcD}$ or both were mixed with prFMN and $\mathrm{MnCl}_{2}$ in a 1:2:2 (protein: $\operatorname{prFMN}: \mathrm{MnCl}_{2}$ ) in an anaerobic chamber and were added to substrate to a final concentration of $10 \mu \mathrm{M}$ protein (both $\mathrm{VdcC}$ and $\mathrm{VdcD} 10 \mu \mathrm{M}$ when added in combination), and diluted to a final substrate concentration of $5 \mathrm{mM}$. The reaction was allowed to proceed overnight $(18 \mathrm{~h})$ in the anaerobic chamber before being removed to aerobic conditions and quenched by the addition of 1 volume equivalent of acetonitrile $+0.2 \%$ trifluoroacetic acid (TFA). The samples were centrifuged at $14,000 \times g$ for $10 \mathrm{~min}$ to removed precipitated protein. The resulting supernatant was diluted 2.5 fold before being subjected to HPLC in isocratic $50 \%$ acetonitrile, $50 \%$ water, $0.1 \%$ TFA on a Phenomenex Kinetex $5 \mu \mathrm{m}$ C18 $100 \AA 250 \times 4.6 \mathrm{~mm} \mathrm{LC}$ Column. Peaks were identified by running standards of substrate and expected product and quantified by running a standard concentration curve of these compounds. A control of substrate with $20 \mu \mathrm{M}$ prFMN was made to ensure there were no side reactions arising from the prFMN reconstitution mix. Samples were run in duplicate.

Assays for the substrate scope experiments were set up in an identical manner with the exception of adding a 2 -fold excess of $\mathrm{VdcD}$ to $\mathrm{VdcC}$ in cross-activation studies. In all assays the concentration of the $\mathrm{C}$ subunit was maintained at $10 \mu \mathrm{M}$.

Data were analysed and peaks integrated using Agilent Chemstation (Version (B.04.03)

VdcC:VdcD Stoichiometry effect assays. The effect of varied stoichiometry was investigated by the anaerobic reconstitution of $S h \mathrm{VdcC}$ in the presence of 0.2 and 4 molar equivalents of $S h \mathrm{VdcD}$. $S h \mathrm{VdcC}$ and $S h \mathrm{VdcD}$ were mixed with prFMN/ $\mathrm{MnCl}_{2}$, giving constant concentrations of $50 \mu \mathrm{M}$ and $200 \mu \mathrm{M}$ for ShVdcC and prFMN/MnCl ${ }_{2}$ respectively, and a concentration of either $10 \mu \mathrm{M}$ or $200 \mu \mathrm{M}$ for ShVdcD. The activity was assayed in anoxic conditions on a Cary $60 \mathrm{UV}-\mathrm{vis}$ Spectrophotometer (Agilent) using the Kinetics software (Version 5.0.0.999) measuring the change in absorbance at $247 \mathrm{~nm}$ using $100 \mu \mathrm{M}$ PHB, and adding $\mathrm{VdcC}$ to a concentration of $0.25 \mu \mathrm{M}$. Substrate was not purged of oxygen prior to activity assays. The measurements were taken over a period of $3 \mathrm{~h}$ in triplicate.

Aerobic decay. To measure the aerobic activity decay, $S h \mathrm{VdcCD}$ was first rendered anaerobic by desalting into $20 \mathrm{mM}$ Hepes, $150 \mathrm{mM} \mathrm{KCl}, \mathrm{pH} 7.5$ in an anaerobic chamber using a GE Healthcare PD-10 Mini desalting column. prFMN (produced as described above) and $\mathrm{MnCl}_{2}$ were added in 2 fold excess. The mixture was left for 10 min prior to desalting into the same buffer. A total of $100 \mu \mathrm{L}$ of active protein was removed from the anaerobic environment in an opaque tube (to ensure no light mediated effects) and the activity was measured every 15 min over a $90 \mathrm{~min}$ period using $100 \mu \mathrm{M}$ PHB in $50 \mathrm{mM} \mathrm{NaPi}, 50 \mathrm{mM} \mathrm{KCl}$, pH 6, measuring the decrease in absorbance at $247 \mathrm{~nm}$ in a Agilent Cary 60 Spectrophotometer using the Kinetics software (Version 5.0.0.999). The protein was constantly exposed to oxygen by leaving the tube open throughout the time course. After 90 minutes the remaining protein was reintroduced to the anaerobic chamber, and a 2 -fold excess of prFMN was added. The protein was removed from the chamber, and immediately assayed for activity. The process was performed in duplicate.

Kinetic solvent viscosity effects (KSVEs). To measure KSVEs, PHB was dissolved in $50 \mathrm{mM} \mathrm{NaPi}, 50 \mathrm{mM} \mathrm{KCl}, \mathrm{pH} 6$, and added to stock solutions of buffered viscosogen $(50 \mathrm{mM} \mathrm{NaPi}, 50 \mathrm{mM} \mathrm{KCl}, \mathrm{pH}$ 6) to a final PHB concentration of $10 \mathrm{mM}$, and viscosogen concentrations of $45 \%$ glycerol and $40 \%$ sucrose. The $\mathrm{pH}$ of $\mathrm{PHB} /$ viscosogen stock solutions were measured to ensure no variation from $\mathrm{pH}$ 6. The PHB stocks were mixed with buffered viscosogen to generate the dilution series required for obtaining kinetic parameters.

prFMN was produced in vitro as described above, and was added to $S h \mathrm{VdcCD}$ in a substoichiometric ratio with excess $\mathrm{MnCl}_{2}\left(0.5 \mathrm{prFMN}\right.$ : 1 ShVdcCD: $2 \mathrm{MnCl}_{2}$ ). This was performed so the final concentration of prFMN (assuming complete turnover in the UbiX reaction) can be considered $\mathrm{E}_{\mathrm{t}}$ for calculations (i.e. [active protein $]=[\mathrm{prFMN}])$. The mixture was diluted to $\mathrm{E}_{\mathrm{t}}^{\mathrm{app}}=30 \mu \mathrm{M}$ with a mixture of aerobic and anaerobic buffers (1:3 ratio) and left overnight in the anaerobic chamber to allow full oxidative maturation in trace oxygen.

Rates were measured using the decrease in absorbance at $247 \mathrm{~nm}$ in an Agilent Cary 60 Spectrophotometer in the Kinetics software (Version 5.0.0.999), using Eppendorf UVette ${ }^{\varpi}$ cuvettes. While measuring rates at $[\mathrm{PHB}] \leq 100 \mu \mathrm{M}$, a $1 \mathrm{~cm}$ pathlength was used; at $\geq 250 \mu \mathrm{M}$ a $0.2 \mathrm{~cm}$ pathlength was used to allow absorbance in the linear range. ShVdcCD prFMN was added to $100 \mu \mathrm{L}$ PHB and agitated vigorously.

Crystallography. Crystal trials were attempted with both $B s \mathrm{VdcCD}$ and $S h \mathrm{VdcCD}$ following purification. Trials were produced in Molecular Dimensions' PACTPremier, JCSG-Plus, Morpheus, SG1, LMB, and BCS screens. Using a TTP Labtech Mosquito, protein was mixed in a 1:1 ratio with reagent, in $600 \mathrm{~nL}$ sitting drops with $30 \mu \mathrm{L}$ reservoir solution. Three concentrations of protein were screened in SWISSCI 3 Lens plates, at 20,10 , and $5 \mathrm{mg} \mathrm{mL}^{-1}$. BsVdcCD was found to crystallise in a number of conditions, however, diffraction was insufficient. ShVdcCD was found to crystallise in multiple conditions, with the best diffracting crystals found in JCSG-Plus D3 (0.2 M Sodium chloride, $0.1 \mathrm{M}$ sodium/potassium phosphate, $\mathrm{pH}$ 6.2, 50\% v/v PEG200) (F222-Closed), BCS H2 (0.1 M Hepes, pH 7.5, $30 \%$ v/v PEG Smear low) (P1-Open), and JCSG-Plus B8 (0.2 M Magnesium chloride hexahydrate, $0.1 \mathrm{M}$ Tris, $\mathrm{pH} 7,10 \%$ PEG 8000) ( $\left.\mathrm{P}_{1} 2_{1} 2_{1}-S h \mathrm{VdcCD}{ }^{\mathrm{V} 99 \mathrm{X}}\right)$. 
The ShVdcCD $559 \mathrm{X}$ crystal was cryoprotected with $10 \%$ PEG 400 prior to flash cooling in liquid $\mathrm{N}_{2}$. F222 and P1 wild type ShVdcCD crystals were flash cooled and required no further cryoprotecting due to components in the crystallisation conditions. Data were collected on Diamond Light source beamlines i03 (F222), i04-1 (P1), and i24 (V59X) and processed with xia244 (P1 and F222) or xia2 dials ${ }^{45}\left(\mathrm{P}_{1} 2_{1} 2_{1}\right)$ using beamline automated data processing. Structures were solved using molecular replacement, using Phaser $\mathrm{MR}^{46}$, with UbiD as a search model. Two ensembles were used to solve the structure, firstly the hexameric core of the oligomerisation domains was used as a single search model (UbiD residues 334-465 arranged in the hexamer), followed by the search for six prFMN binding domains (UbiD residues 1-333). The F222 model was initially built using phenix.autobuild ${ }^{47}$, prior to manual model building in $\operatorname{Coot}^{48}$. The model was refined using phenix.refine ${ }^{49}$, interspersed with manual model building. The closed model was then used in molecular replacement of the open structures (P1, $\mathrm{P} 22_{1} 2_{1}$ ), using the same strategy as was used for solving the closed structure. The models were then refined and built using phenix.refine and coot. The models and data were deposited in the PDB under the accession codes (Closed-7AE4, Open-7AE5, ShVdcCD ${ }^{\mathrm{V} 59 \mathrm{X}}$-7AE7). Data processing statistics, along with refinement statistics are in Table 1.

Modelling and docking. The prFMN binding domains of the two states were aligned on secondary structure, prior to a Morph being calculated using UCSF Chimera $1.13 .1^{37}$. Analysis showed that the largest motions were from the closure of the domains, however, loop motions around the prFMN binding pocket were observed, with the closed state closely resembling crystal structures in which prFMN can be seen binding. Therefore, a hybrid model was constructed, with the prFMN binding domain (residues 1-323) of the closed structure being used in conjunction with the oligomerisation domain of the open structure to create an open model with the prFMN binding pocket in an appropriate conformation. A new morph was performed, with the trajectory of the oligomerisation domain being plotted in 16 models, allowing an approximate $2^{\circ}$ iterations of the motion. Cartesian coordinates for the Int 2 and Int 3 Quinoid intermediates with the simplest substrate (PHB) were built from previous DFT models of prFMN-substrate adducts ${ }^{17}$ and were optimised using Gaussian 09 revision D.01 at the B3LYP/6$311++\mathrm{G}(\mathrm{d}, \mathrm{p})$ level of theory with the D3 version of Grimme's dispersion with Becke-Johnson damping ${ }^{50}$ and a generic polarizable continuum model with a dielectric of 5.7. These intermediates were docked into the 16 morph models using AutoDock Vina ${ }^{38}$. To ensure only the effect of domain closure was being observed, all residues were restricted to a single conformer.

Substrate and product docking was performed using the coordinates described above, with prFMN present in the position as determined by Rosetta Ligand Docking ${ }^{35}$. For PHB docking E278 was allocated an allowed rotamer to prevent occlusion of the substrate carboxylate binding pocket.

Docking of Int 2 and Int 3 Quinoid resulted in single binding poses in each of the sixteen models. Where substrates and product were docked, multiple poses were observed. In these instances, the pose with the lowest energy for the correct orientation (of substrate/product carboxy and hydroxy groups based on prior knowledge) was selected to resemble a likely catalytically relevant species.

Models and ligand co-ordinates used for docking are available at https://doi.org/ 10.5281/zenodo.5101186, along with binding energies calculated by Autodock Vina.

EINémo was used to assess the low frequency normal mode analysis of domain motion from crystal structures. Both open and closed structures were analysed. Parameters were set as default, with the exception of $\mathrm{NRBL}=5$, and DQMIN $=-200$, DQMAX $=200$

Table 1 X-ray crystallography data collection and refinement statistics.

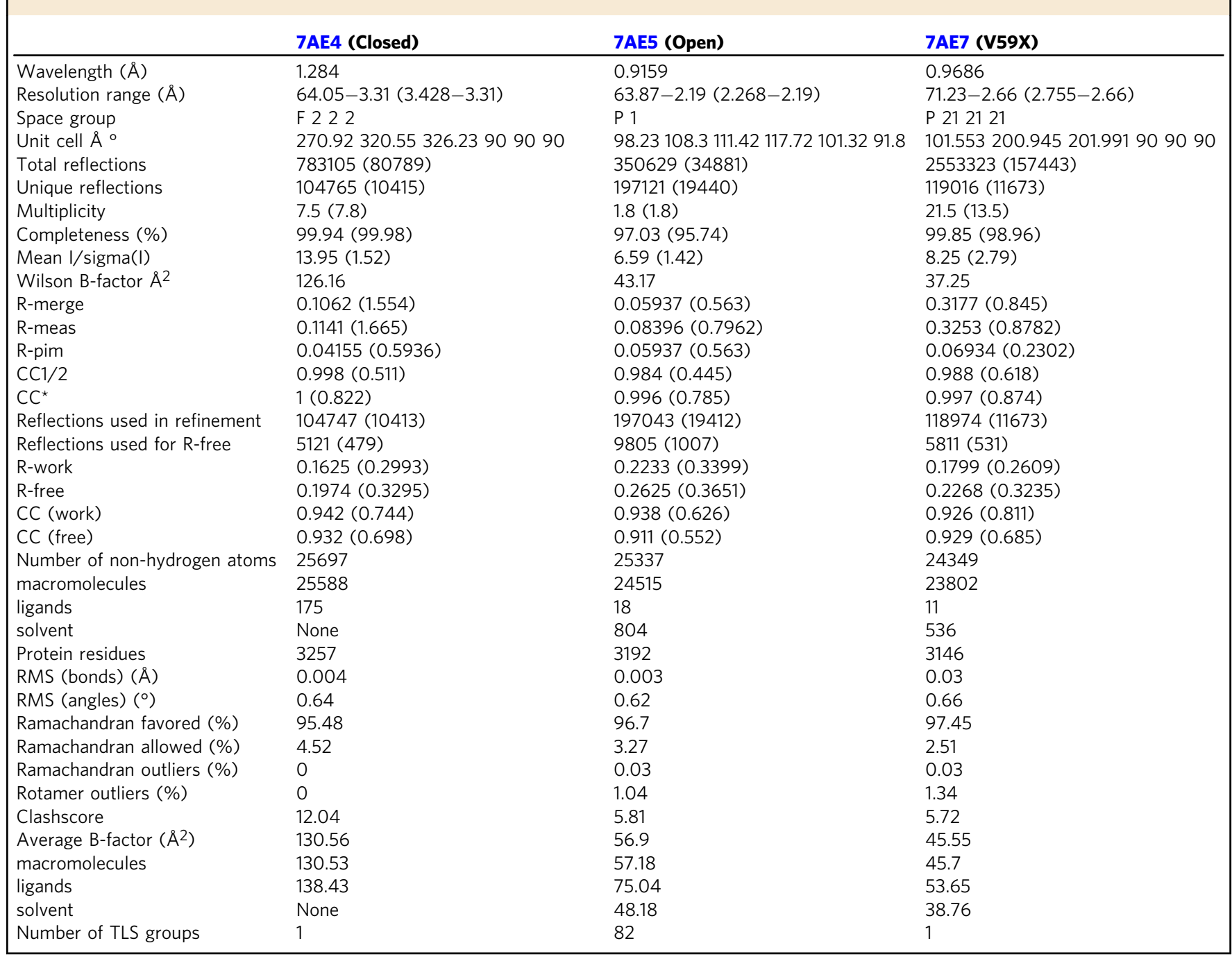


Reporting summary. Further information on research design is available in the Nature Research Reporting Summary linked to this article.

\section{Data availability}

The atomic coordinates and structures factors (codes 7AE4, 7AE5, and 7AE7) have been deposited to the Protein Data Bank (http://www.pdb.org). Graphical data presented here is available in the source data file. Modelling data is available at https://doi.org/10.5281/ zenodo.5101186. Publicly available data used in this study is available from the Protein Data Bank under the accession codes: $4 \mathrm{ZA} 4,5 \mathrm{O} 3 \mathrm{M}, 5 \mathrm{O} 3 \mathrm{~N}, 5 \mathrm{NY} 5,5 \mathrm{M} 1 \mathrm{C}, 5 \mathrm{M} 1 \mathrm{D}, 6 \mathrm{H} 6 \mathrm{~V}$, 6DA6, 6DA7, 6DA9, 7ABN Source data are provided with this paper.

Received: 3 December 2020; Accepted: 20 July 2021;

Published online: 20 August 2021

\section{References}

1. Becker, J. \& Wittmann, C. A field of dreams: Lignin valorization into chemicals, materials, fuels, and health-care products. Biotechnol. Adv. 37, 107360 (2019).

2. Werpy, T. \& Petersen, G. Top value added chemicals from biomass: volume I-results of screening for potential candidates from sugars and synthesis gas. (National Renewable Energy Lab., Golden, CO (US), 2004).

3. Llevot, A., Grau, E., Carlotti, S., Grelier, S. \& Cramail, H. From lignin-derived aromatic compounds to novel biobased polymers. Macromol. Rapid Commun. 37, 9-28 (2016).

4. Eerhart, A., Faaij, A. \& Patel, M. K. Replacing fossil based PET with biobased PEF; process analysis, energy and GHG balance. Energy Environ. Sci. 5, 6407-6422 (2012).

5. Aleku, G. A. et al. Enzymatic C-H activation of aromatic compounds through $\mathrm{CO}_{2}$ fixation. Nat. Chem. Biol. 16, 1255-1260 (2020)

6. Payne, K. A. P. et al. Enzymatic carboxylation of 2-furoic acid yields 2,5furandicarboxylic acid (FDCA). ACS Catal. 9, 2854-2865 (2019).

7. Kawanabe, K., Aono, R. \& Kino, K. 2, 5-Furandicarboxylic acid production from furfural by sequential biocatalytic reactions. J. Biosci. Bioeng. 132, 18-24 (2021).

8. Weber, H. E. et al. Requirement of a functional FMN prenyltransferase for the activity of a bacterial decarboxylase in a heterologous muconic acid pathway in Saccharomyces cerevisiae. Appl. Environ. Microbiol. 83, 03472-16 (2017).

9. Jensen, E. D. et al. Integrating continuous hypermutation with highthroughput screening for optimization of cis, cis-muconic acid production in yeast. Microbial Biotechnol. (2021).

10. Mori, Y., Noda, S., Shirai, T. \& Kondo, A. Direct 1, 3-butadiene biosynthesis in Escherichia coli via a tailored ferulic acid decarboxylase mutant. Nat. Commun. 12, 1-12 (2021).

11. White, M. D. et al. UbiX is a flavin prenyltransferase required for bacterial ubiquinone biosynthesis. Nature 522, 502-506 (2015).

12. Marshall, S. A., Payne, K. A. \& Leys, D. The UbiX-UbiD system: the biosynthesis and use of prenylated flavin (prFMN). Arch. Biochem. Biophys. 632, 209-221 (2017).

13. Meckenstock, R. U. et al. Anaerobic degradation of benzene and polycyclic aromatic hydrocarbons. J. Mol. Microbiol. Biotechnol. 26, 92-118 (2016).

14. Abu Laban, N., Selesi, D., Rattei, T., Tischler, P. \& Meckenstock, R. U. Identification of enzymes involved in anaerobic benzene degradation by a strictly anaerobic iron-reducing enrichment culture. Environ. Microbiol. 12, 2783-2796 (2010).

15. Bergmann, F. D., Selesi, D. \& Meckenstock, R. U. Identification of new enzymes potentially involved in anaerobic naphthalene degradation by the sulfate-reducing enrichment culture N47. Arch. Microbiol. 193, 241-250 (2011).

16. Payne, K. A. et al. New cofactor supports alpha,beta-unsaturated acid decarboxylation via 1,3-dipolar cycloaddition. Nature 522, 497-501 (2015).

17. Bailey, S. S. et al. Enzymatic control of cycloadduct conformation ensures reversible 1,3-dipolar cycloaddition in a prFMN-dependent decarboxylase. Nat. Chem. 11, 1049-1057 (2019).

18. Payer, S. E. et al. Regioselective para-carboxylation of catechols with a prenylated flavin dependent decarboxylase. Angew. Chem. Int. Ed. 56, 13893-13897 (2017).

19. Payne, K. A. et al. Structure and mechanism of Pseudomonas aeruginosa PA0254/HudA, a prFMN-dependent pyrrole-2-carboxylic acid decarboxylase linked to virulence. ACS Catal. 11, 2865-2878 (2021).

20. Marshall, S. A. et al. Oxidative maturation and structural characterization of prenylated-FMN binding by UbiD, a decarboxylase involved in bacterial ubiquinone biosynthesis. J. Biol. Chem. 292, 4623-4637 (2017).
21. Lupa, B., Lyon, D., Shaw, L. N., Sieprawska-Lupa, M. \& Wiegel, J. Properties of the reversible nonoxidative vanillate/4-hydroxybenzoate decarboxylase from Bacillus subtilis. Can. J. Microbiol. 54, 75-81 (2008).

22. Schuhle, K. \& Fuchs, G. Phenylphosphate carboxylase: a new C-C lyase involved in anaerobic phenol metabolism in Thauera aromatica. J. Bacteriol. 186, 4556-4567 (2004).

23. Jimenez, N., Curiel, J. A., Reveron, I., de Las Rivas, B. \& Munoz, R. Uncovering the Lactobacillus plantarum WCFS1 gallate decarboxylase involved in tannin degradation. Appl. Environ. Microbiol. 79, 4253-4263 (2013).

24. He, Z. \& Wiegel, J. Purification and characterization of an oxygen-sensitive reversible 4-hydroxybenzoate decarboxylase from Clostridium hydroxybenzoicum. Eur. J. Biochem. 229, 77-82 (1995).

25. Chow, K. T., Pope, M. K. \& Davies, J. Characterization of a vanillic acid nonoxidative decarboxylation gene cluster from Streptomyces sp. D7. Microbiology 145(Pt 9), 2393-2403 (1999).

26. Lupa, B., Lyon, D., Gibbs, M. D., Reeves, R. A. \& Wiegel, J. Distribution of genes encoding the microbial non-oxidative reversible hydroxyarylic acid decarboxylases/phenol carboxylases. Genomics 86, 342-351 (2005).

27. Mergelsberg, M. et al. Phthaloyl-coenzyme A decarboxylase from Thauera chlorobenzoica: the prenylated flavin-, $\mathrm{K}+$-and Fe2+-dependent key enzyme of anaerobic phthalate degradation. Environ. Microbiol. 19, 3734-3744 (2017)

28. Krishna, S. S., Majumdar, I. \& Grishin, N. V. Structural classification of zinc fingers: survey and summary. Nucleic Acids Res. 31, 532-550 (2003).

29. Krissinel, E. \& Henrick, K. Secondary-structure matching (SSM), a new tool for fast protein structure alignment in three dimensions. Acta Crystallogr. Sect. D: Biol. Crystallogr. 60, 2256-2268 (2004)

30. Qian, X. et al. Novel zinc finger motif in the basal transcriptional machinery: three-dimensional NMR studies of the nucleic acid binding domain of transcriptional elongation factor TFIIS. Biochemistry 32, 9944-9959 (1993).

31. Krissinel, E. \& Henrick, K. Inference of macromolecular assemblies from crystalline state. J. Mol. Biol. 372, 774-797 (2007).

32. Bailey, S. S. et al. The role of conserved residues in Fdc decarboxylase in prenylated flavin mononucleotide oxidative maturation, cofactor isomerization, and catalysis. J. Biol. Chem. 293, 2272-2287 (2018).

33. Poornam, G. P., Matsumoto, A., Ishida, H. \& Hayward, S. A method for the analysis of domain movements in large biomolecular complexes. Proteins: Struct. Funct. Bioinforma. 76, 201-212 (2009).

34. Costantini, S., Paladino, A. \& Facchiano, A. M. CALCOM: a software for calculating the center of mass of proteins. Bioinformation 2, 271 (2008).

35. DeLuca, S., Khar, K. \& Meiler, J. Fully flexible docking of medium sized ligand libraries with RosettaLigand. PLOS ONE 10, e0132508 (2015).

36. Lyskov, S. et al. Serverification of molecular modeling applications: the Rosetta Online Server that Includes Everyone (ROSIE). PLoS ONE 8, e63906 (2013).

37. Pettersen, E. F. et al. UCSF Chimera-a visualization system for exploratory research and analysis. J. Comput. Chem. 25, 1605-1612 (2004).

38. Trott, O. \& Olson, A. J. AutoDock Vina: improving the speed and accuracy of docking with a new scoring function, efficient optimization, and multithreading. J. Comput. Chem. 31, 455-461 (2010).

39. Suhre, K. \& Sanejouand, Y.-H. ElNemo: a normal mode web server for protein movement analysis and the generation of templates for molecular replacement. Nucleic acids Res. 32, W610-W614 (2004).

40. Gadda, G. \& Sobrado, P. Kinetic solvent viscosity effects as probes for studying the mechanisms of enzyme action. Biochemistry 57, 3445-3453 (2018).

41. Jacewicz, A., Izumi, A., Brunner, K., Schnell, R. \& Schneider, G. Structural insights into the UbiD protein family from the crystal structure of PA0254 from Pseudomonas aeruginosa. PLoS ONE 8, 10 (2013).

42. Marshall, S. A. et al. Heterologous production, reconstitution and EPR spectroscopic analysis of prFMN dependent enzymes. Methods Enzymol. 620, 489-508 (2019).

43. Shepherd, S. A. et al. Extending the biocatalytic scope of regiocomplementary flavin-dependent halogenase enzymes. Chem. Sci. 6, 3454-3460 (2015).

44. Winter, G. xia2: an expert system for macromolecular crystallography data reduction. J. Appl. Crystallogr. 43, 186-190 (2009).

45. Winter, G. et al. DIALS: implementation and evaluation of a new integration package. Acta Crystallogr. Sect. D. 74, 85-97 (2018).

46. McCoy, A. J. Solving structures of protein complexes by molecular replacement with Phaser. Acta Crystallogr. Sect. D. Biol. Crystallogr. 63, 32-41 (2007).

47. Terwilliger, T. C. et al. Iterative model building, structure refinement and density modification with the PHENIX AutoBuild wizard. Acta Crystallogr. Sect. D: Biol. Crystallogr. 64, 61-69 (2008).

48. Emsley, P. \& Cowtan, K. Coot: model-building tools for molecular graphics. Acta Crystallogr. Sect. D. Biol. Crystallogr. 60, 2126-2132 (2004).

49. Afonine, P. V. et al. Towards automated crystallographic structure refinement with phenix.refine. Acta Crystallogr. Sect. D. Biol. Crystallogr. 68, 352-367 (2012).

50. Grimme, S., Ehrlich, S. \& Goerigk, L. Effect of the damping function in dispersion corrected density functional theory. J. Comput. Chem. 32, 1456-1465 (2011) 
51. Hay, S., Pudney, C. R., Sutcliffe, M. J. \& Scrutton, N. S. Are environmentally coupled enzymatic hydrogen tunneling reactions influenced by changes in solution viscosity? Angew. Chem. Int. Ed. 47, 537-540 (2008).

\section{Acknowledgements}

We would like to thank Diamond Light Source for access to beamlines under the proposals MX12788 and MX17773. This work was supported by the ERC grant pre-FAB ADG_695013 and BBSRC grant BB/P000622/1, D.L. is a Royal Society Wolfson Research Merit Award holder.

\section{Author contributions}

D.L. conceived the study and together with S.A.M. coordinated the work. S.A.M. carried out experiments with help from K.A.P.P. (molecular biology), K.F. (protein expression/ purification), G.R.T. (KSVE data acquisition), C. L. (modelling), and S.H. (computational studies). S.A.M. and D.L. wrote the paper. All authors reviewed the results and approved the final version of the manuscript.

\section{Competing interests}

The authors declare no competing interests.

\section{Additional information}

Supplementary information The online version contains supplementary material available at https://doi.org/10.1038/s41467-021-25278-z.
Correspondence and requests for materials should be addressed to S.A.M. or D.L.

Peer review information Nature Communications thanks Michael Torrens-Spence and the other anonymous reviewer(s) for their contribution to the peer review of this work.

Reprints and permission information is available at http://www.nature.com/reprints

Publisher's note Springer Nature remains neutral with regard to jurisdictional claims in published maps and institutional affiliations.

(c) (i) Open Access This article is licensed under a Creative Commons Attribution 4.0 International License, which permits use, sharing, adaptation, distribution and reproduction in any medium or format, as long as you give appropriate credit to the original author(s) and the source, provide a link to the Creative Commons license, and indicate if changes were made. The images or other third party material in this article are included in the article's Creative Commons license, unless indicated otherwise in a credit line to the material. If material is not included in the article's Creative Commons license and your intended use is not permitted by statutory regulation or exceeds the permitted use, you will need to obtain permission directly from the copyright holder. To view a copy of this license, visit http://creativecommons.org/ licenses/by/4.0/.

(C) The Author(s) 2021 